\section{RÉFÉRENCES}

1. Khalife M, Young R, Passet B, et al. Transcriptomic analysis brings new insight into the biological role of the prion protein during mouse embryogenesis. PLOS One $2011 ; 6$ : e23253.

2. Malaga-Trillo $\varepsilon$, Solis GP, Schrock $y$, et al. Regulation of embryonic cell adhesion by the prion protein. PLOS Biol $2009 ; 7$ : e55.

3. Caughey B, Baron GS, Chesebro B, Jeffrey M. Getting a grip on prions: oligomers, amyloids, and pathologica membrane interactions. Annu Rev Biochem 2009; 78 : 177-204.

4. Collinge J, Clarke AR. A general model of prion strains and their pathogenicity. Science 2007 ; 318 : 930-6.
5. Tixador P, Herzog L, Reine F, et al. The physical relationship between infectivity and prion protein aggregates is strain-dependent. PLoS Pathog 2010 6: el000859.

6. Beringue V, Vilotte JL, Laude H. Prion agent diversity and species barrier. Vet Res 2008 ; $39: 47$.

7. Beringue V, Herzog L, Jaumain $\varepsilon$, et al. Facilitated cross-species transmission of prions in extraneural tissue. Science $2012 ; 335$ : 472-5

8. Asante $\varepsilon A$, Linehan JM, Desbruslais M, et al. BSE prions propagate as either variant CJD-like or sporadic CJD-like prion strains in transgenic mice expressing human prion protein. EMBO J 2002 ; 21 : 6358-66.

NOUVELLE

\title{
Un transporteur du magnésium
}

\section{Nouvel intermédiaire dans les voies de signalisation du TCR}

Gilles Besin ${ }^{1,2}$

> Les ions magnésium $\left(\mathrm{Mg}^{2+}\right)$ sont les cations les plus abondants dans les cellules de mammifères et constituent un cofacteur essentiel pour l'ATP, les acides nucléiques, et de nombreuses enzymes chez les animaux et les plantes $[1,2]$. Toutefois, contrairement aux ions calcium $\left(\mathrm{Ca}^{2+}\right)$ pour lesquels les concentrations intracellulaires d'ions libres $\left(\left[\mathrm{Ca}^{2+}\right]_{\text {Int }}\right.$ environ $\left.0,1 \mathrm{mM}\right)$ et les concentrations extracellulaires $\left(\left[\mathrm{Ca}^{2+}\right]_{e x}\right.$ environ $1 \mathrm{mM}$ ) permettent des mouvements et flux ioniques, aucune étude n'a démontré clairement le rôle des ions $\mathrm{Mg}^{2+}$ en tant que messagers secondaires dans la signalisation intracellulaire. Les ions magnésium intracellulaires $\left(\left[\mathrm{Mg}^{2+}\right]_{\text {Int }}\right.$ environ $20 \mathrm{mM}$ ) sont surtout complexés avec I'ATP, I'ADN, des enzymes ou d'autres molécules, et seulement 1 à $5 \%$ de ces ions magnésium cellulaires (0,2-1 mM) sont sous une forme libre dans le cytosol. Fait intéressant, dans diverses études, I'utilisation de sondes sensibles aux flux d'ions magnésium a permis de démontrer des changements dans la concentration intracellulaire de ces ions dans certaines cellules du système immunitaire comme les lymphocytes (après stimulation par des lectines) [3-5].

Le système immunitaire représente la principale défense contre les infections virales, bactériennes ou les cancers, et tout défaut du système immunitaire entraîne une sensibilité à ces agressions pouvant avoir de graves conséquences. Le décryptage moléculaire de multiples déficits immunitaires primaires révèle souvent de nouveaux acteurs intervenant dans l'activation lymphocytaire. Ainsi, en étudiant trois patients immunodéficients, l'équipe de M. Lenardo (National institutes of health [NIH], Bethesda, États-Unis) a identifié récemment le Magnesium tranporter protein I (MAGTl) comme un nouveau maillon de la voie d'activation des lymphocytes T [6].

L'activation des lymphocytes $T$ est déclenchée par l'interaction entre le récepteur des lymphocytes T (TCR) et une molécule du complexe majeur d'histocompatibilité ( $C M H$ ) chargée d'un peptide antigénique, exprimée à la surface d'une cellule présentatrice d'antigènes ( $A P C)$. Cet engagement $d u$ TCR entraîne l'activation des enzymes
9. Bishop MT, Hart P, Aitchison L, et al. Predicting susceptibility and incubation time of human-tohuman transmission of vCJD. Lancet Neurol $2006 ; 5$ : 393-8.

10. Health Protection Agency, UK. http://www.hpa.org uk/hpr/archives/2011/news3611.htm.

11. Hilton DA, Ghani AC, Conyers L, et al. Prevalence of lymphoreticular prion protein accumulation in UK tissue samples. J Pathol 2004 ; 203 : 733-9.

12. Bachy V, Aucouturier P. Maladies à prions. Quel rôle pour les cellules dendritiques dans la pathogenèse des formes transmises? Med Sci (Paris) $2010 ; 26$ : 615-20.
${ }^{1}$ In-Cell-Art ; ${ }^{2}$ Institut du thorax, UMR 1087 Inserm/UMR 6291 CNRS/IRT-UN, 8, quai Moncousu, 44007 Nantes Cedex 1, France.

Gilles.Besin@univ-nantes.fr

kinases Lck, ZAP-70 (zeta-chain-associated protein kinase of $70 \mathrm{kDa}$ ) et Itk (IL-2 inducible T-cell kinase) qui phosphorylent la protéine PLC $\gamma \mathrm{l}$ (phospholipase $(\gamma)$ ainsi que d'autres protéines. La phosphorylation de la PLCy entraîne l'augmentation de la concentration intracellulaire en ions calcium $\left(\left[\mathrm{Ca}^{2+}\right]_{\text {Int }}\right)$ et l'activation de plusieurs facteurs de transcription: NFAT (nuclear factor of activated $T$-cells), NF- $\kappa \mathrm{B}$ (nuclear factor kappa B), etc. Des mutations de ces molécules clés de l'activation de la voie du TCR (Lck, ZAP-70, Itk, LAT [linker of activation of $T$ cell], PLC $\gamma \mathrm{l}$ ) ont été identifiées chez plusieurs patients immunodéficients [7].

\section{Un nouveau déficit immunitaire lié à l'X}

Le groupe de $M$. Lenardo a étudié trois patients issus de deux familles différentes. Le déficit immunitaire de ces patients était classé comme lymphopénie CD4 idiopathique $(I C L)^{1}$ et

\footnotetext{
${ }^{1}$ Défini par un taux de $\mathrm{CD} 4^{+}<300 / \mathrm{mm}^{3}$ ou $<20 \%$ du total de lymphocytes en l'absence d'infection par le VIH ou d'autres causes évidentes de lymphopénie.
} 
caractérisé par des taux bas de lymphocytes $\mathrm{T} \mathrm{CD}^{+}$, alors que les autres populations de lymphocytes étaient présentes en nombre normal ou élevé et que les taux d'immunoglobulines et les réponses vaccinales n'étaient anormales que de façon intermittente. Le rapport des lymphocytes $\operatorname{CD} 4^{+} / \mathrm{CD} 8^{+}$ était inversé et une réduction des cellules $\mathrm{CD} \mathrm{I}^{+}$au sein de la population des lymphocytes T CD4 $4^{+}$naïfs suggérait une diminution de la production thymique. L'activation des lymphocytes T en réponse au signal du TCR ou à un anti-CD3 agoniste était très anormale et le défaut a été localisé précisément à une étape précoce de la cascade d'activation. La réponse immunitaire humorale était normale. Cliniquement, ces patients étaient atteints d'infections récurrentes mal contrôlées, mais essentiellement virales, en particulier par le virus d'Epstein-Barr.

Orientés par l'atteinte de deux garçons dans une des deux familles et un biais d'inactivation du chromosome $X$ chez la mère, les auteurs ont suspecté une atteinte liée à I'X. Celle-ci a été confirmée par séquençage haut débit et capture d'exon, qui ont mis en évidence une délétion de 10 paires de bases dans le gène MAGTI des deux frères; elle était absente chez la mère, mais présente chez la grand-mère et l'arrière grandmère des 2 patients. Cette délétion supprime un site donneur d'épissage situé à la jonction exon-intron de l'exon 7. Les transcrits sont effondrés et la protéine indétectable. Chez le troisième patient, il s'agissait d'une mutation non-sens de I'exon 3 de MAGTI.

\section{Implication fonctionnelle du gène MAGTI}

Le gène MAGTI code pour un transporteur membranaire du magnésium, MAGTl, dont les fonctions physiologiques ne sont pas encore totalement élucidées. Alors que la stimulation du TCR induit une entrée massive des ions $\mathrm{Mg}^{2+}$ dans les cellules T normales [3-5], cet influx est indétectable dans les lymphocytes $\mathrm{T}$ des patients. Une analyse précise a montré que ce défaut était très spécifique : il n'affectait pas les processus métaboliques cellulaires dont le $\mathrm{Mg}^{2+}$ est un cofacteur, mais intervenait uniquement sur les événements d'activation en aval de la phosphorylation de la protéine $\mathrm{PLC} \gamma \mathrm{l}$ qui est diminuée dans les lymphocytes $T$ des patients. Les fonctions des lymphocytes $B$, qui expriment le gène MAGTI, n'étaient pas touchées chez ces patients par l'absence de fonction du transporteur MAGTl (mais c'est la PLC $\gamma 2$ qui est impliquée dans l'acti-

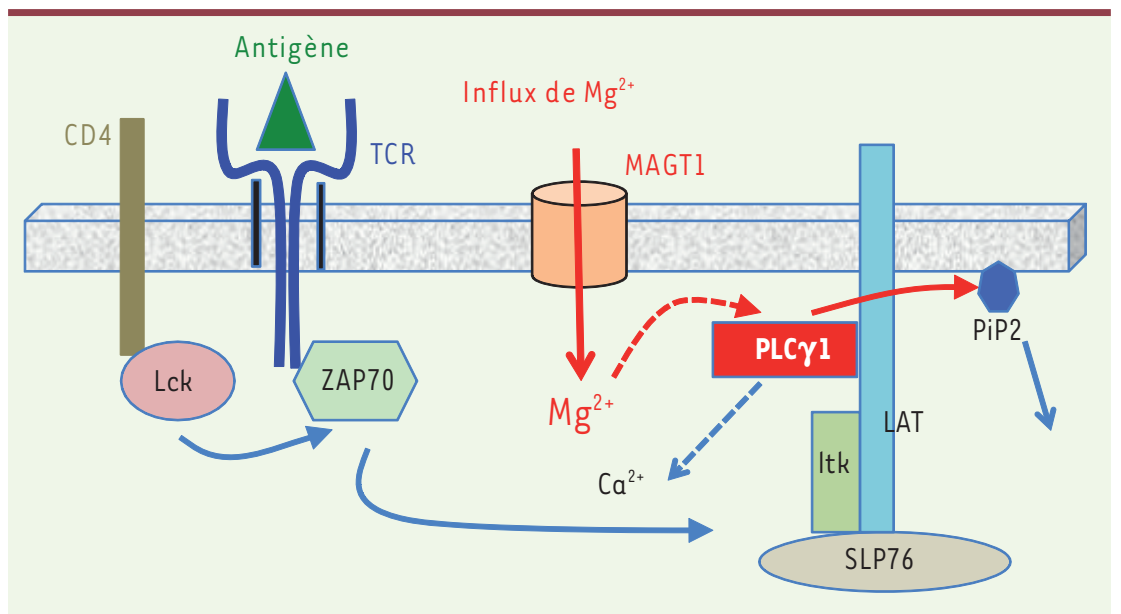

Figure 1. Localisation de l'action de l'influx de $\mathrm{Mg}^{2+}$ relayée par le transporteur MAGTI dans l'activation lymphocytaire $T$ (adapté de [10]).

vation du récepteur des lymphocytes $B$ $[B C R])$. Des expériences complémentaires in vitro de délétion transitoire du gène MAGTI dans des lymphocytes normaux, par ARN interférence, et de restauration de l'expression du gène MAGTI normal via des vecteurs lentiviraux dans les lymphocytes $T$ de ces patients, ont confirmé le rôle du transporteur MAGTI dans ces défauts d'activation lymphocytaire. Curieusement, cette perte de flux ionique d'ions $\mathrm{Mg}^{2+}$ était associée à une baisse de l'influx de $\mathrm{Ca}^{2+}$ qui, lui, est tout à fait connu lors de l'activation des lymphocytes T. Cette baisse concomitante de l'influx calcique n'est pas complètement expliquée.

Des études antérieures avaient déjà démontré le rôle important des ions $\mathrm{Mg}^{2+}$ dans l'activation des lymphocytes $T[8,9]$. Des influx de magnésium ont aussi été observés dans deux lignées de cellules épithéliales en réponse à l'EGF (epidermal growth factor) qui agit également via la PLC $\gamma$ l. Dans ce modèle aussi, des flux de calcium accompagnent I'influx de $\mathrm{Mg}^{2+}$, comme lors de l'activation des lymphocytes T via le TCR. Cette corrélation entre les deux influx ioniques n'existe pas lorsque d'autres PLC sont impliquées, PLC $\gamma 2$ ou PLC $\beta$.

\section{Conclusion}

Le travail de ce groupe révèle l'importance physiologique du transporteur MAGTl, qui relaie un influx transitoire de $\mathrm{Mg}^{2+}$ déclenché par l'activation d'un récepteur. Cet influx joue un rôle de second messager dans les lymphocytes T activés (via l'activation de la PLC $\gamma l$ et l'influx de $\mathrm{Ca}^{2+}$ ) mais aussi dans les cellules épithéliales en réponse à l'EGF. Comme souvent en immunologie, c'est l'exploration d'un déficit immunitaire qui a permis cette découverte. II faut toutefois démontrer que la diminution de la réponse des lymphocytes $T$ associée à une diminution de la fonction de MAGTl est uniquement due à la baisse des flux de magnésium. II se pourrait que le transporteur MAGTl possède d'autres fonctions importantes, mais 
non encore identifiées, dans l'activation des lymphocytes $T$.

Enfin, il est curieux que les fonctions des lymphocytes $B$, qui expriment le gène MAGTI, ne semblent pas touchées chez ces patients par l'absence de fonction du transporteur MAGTI. II serait intéressant de compléter l'analyse de l'expression de MAGTl dans les autres cellules du système immunitaire et de déterminer les éventuels défauts de fonction de ces dernières. Enfin, on ne peut exclure que d'autres défauts des lymphocytes $T$ puissent être dus à l'absence de fonction de MAGTI. $\diamond$

Magnesium transporter protein $\mathbf{1}$, a new intermediate in TCR signaling

\section{LIENS D'INTÉRÊT}

L'auteur déclare n'avoir aucun lien d'intérêt concernant les données publiées dans cet article.

\section{RÉFÉRENCES}

1. Cowan JA. Structural and catalytic chemistry of magnesium-dependent enzymes. Biometals 2002 ; $15: 225-35$.

2. Yang W, Lee Jy, Nowotny M. Making and breaking nucleic acids: two- $\mathrm{Mg}^{2+}$-ion catalysis and substrate specificity. Mol Cell $2006 ; 22: 5-13$.

3. Abboud CN, Scully SP, Lichtman AH, et al. The requirements for ionized calcium and magnesium in lymphocyte proliferation.J Cell Physiol 1985 ; 122 : 64-72.

4. Modiano JF, Kelepouris E, Kern JA, Nowell PC. Requirement for extracellular calcium or magnesium in mitogen-induced activation of human peripheral blood lymphocytes. J Cell Physiol 1988 ; 135 : 451-8.

5. Whitney RB, Sutherland RM. The influence of calcium, magnesium and cyclic adenosine 3', 5 '-monophosphate on the mixed lymphocyte reaction. J Immunol $1972 ; 108:$ 1179-83.
6. Li Fy, Chaigne-Delalande B, Kanellopoulou C, et al. Second messenger role for $\mathrm{Mg}^{2+}$ revealed by human T-cell immunodeficiency. Nature 2011 ; 475 : 471-6.

7. Casanova JL, Abel L. Primary immunodeficiencies: a field in its infancy. Science $2007 ; 317: 617-9$.

8. Rijkers GT, Griffioen AW. Changes in free cytoplasmic magnesium following activation of human lymphocytes. Biochem J $1993 ; 289$ : 373-7.

9. Rijkers GT, Henriquez N, Griffioen AW. Intracellular magnesium movements and lymphocyte activation. Magnes Res 1993 ; 6 : 205-13.

10. Wu N, Veillette A. Immunology: magnesium in a signalling role. Nature $2011 ; 475$ : 462-3.

\section{NOUVELLE}

\section{Un nouveau rôle de p27 KIP1 dans la mitose?}

Bérénice Leclercq, Arnaud Besson
Inserm UMR1037, centre de recherche en cancérologie de Toulouse, Toulouse, France ; université de Toulouse, 118, route de Narbonne, 31062 Toulouse Cedex 09, France. CNRS équipe de recherche labellisée ( $\varepsilon R L) 5294$, Toulouse, France. arnaud.besson@inserm.fr p2 $7^{\mathrm{KIP} 1}$ : un inhibiteur des complexes cycline/CDK

La progression dans le cycle de division cellulaire est régie par l'activation séquentielle des complexes cycline/ CDK (cyclin-dependent kinases). Ces complexes sont finement régulés à de multiples niveaux, notamment par des inhibiteurs de CDK dont p27 ${ }^{\mathrm{KIPl}}$ (cyclindependent kinase inhibitor 1B ou p27) [1, 2]. L'importance de p27 dans le contrôle des transitions de GO (quiescence) à $\mathrm{Gl}$, et de Gl à $S$ a été abondamment décrite depuis le clonage du gène codant pour p27 en 1994 [1]. Le niveau de p27 est élevé dans les cellules quiescentes, et diminue lors de la transition Gl/S pour rester faible dans les phases $S, G 2$ et $M$ du cycle cellulaire. La dégradation de p27 est déclenchée par sa phosphorylation par le complexe cycline $\varepsilon /$ CDK2, ce qui crée un site de reconnaissance pour l' $\varepsilon 3$ ubiquitine ligase Skp2 (S-phase kinase-associated protein 2)
[1]. L'invalidation chez la souris du gène cdknlb, qui code pour p27, souligne l'importance de p27 dans le contrôle de la prolifération cellulaire puisque la taille de ces animaux augmente d'environ $30 \%$, et que cette croissance s'accompagne d'une hyperplasie de divers organes et d'une prédisposition à la tumorigenèse spontanée ou induite par des carcinogènes [1]. De plus, la perte de l'expression nucléaire de p27 est un facteur de mauvais pronostic dans de nombreux types de cancers chez l'humain [1]. En outre, la diminution nucléaire de p27 n'est pas associée à des mutations génétiques, comme c'est le cas pour les suppresseurs de tumeurs classiques, mais elle résulte de l'augmentation de sa dégradation protéolytique ou de son exclusion du noyau.

\section{p $27^{\mathrm{KIPl}}$ : protéine multifonctionnelle} De nombreuses études indiquent que le rôle de p27 ne se limite pas à l'inhibition du cycle cellulaire, et que cette protéine participe en fait à la régulation d'autres processus cellulaires via son interaction avec divers partenaires protéiques $[1,2]$. En effet, p27 est impliquée dans le contrôle de la migration cellulaire, de l'apoptose, de la transcription et du devenir des cellules souches progénitrices de p27 [1, 3, 4]. Afin d'étudier les fonctions indépendantes des complexes cycline/CDK, nous avons généré des souris knock-in exprimant un allèle de p27 incapable de lier les cyclines et les CDK $\left(p 27^{C K-}\right)[2,4,5]$. À l'inverse des souris p $27^{-/-}$qui présentent des tumeurs spontanées uniquement au niveau de l'hypophyse, les souris $p 27^{C K-}$ développent des tumeurs dans divers organes dont les poumons. Ce modèle murin a donc révélé un rôle oncogénique pour p27, indépendant de ses fonctions d'inhibiteur de cycline/CDK.

Une autre caractéristique de la protéine $\mathrm{p} 27^{\mathrm{CK}-}$ est qu'en absence de 